\title{
Performance of the SciBar cosmic ray telescope (SciCRT) toward the detection of high-energy solar neutrons in solar cycle 24
}

\author{
Yoshinori Sasai $^{1 *}$, Yuya Nagai ${ }^{1}$, Yoshitaka Itow ${ }^{1}$, Yutaka Matsubara1 , Takashi Sako ${ }^{1}$, Diego Lopez ${ }^{1}$, \\ Tsukasa Itow ${ }^{1}$, Kazuoki Munakata², Chihiro Kato², Masayoshi Kozai², Takahiro Miyazaki², \\ Shoichi Shibata ${ }^{3}$, Akitoshi Oshima ${ }^{3}$, Hiroshi Kojima ${ }^{4}$, Harufumi Tsuchiya ${ }^{5}$, Kyoko Watanabe ${ }^{6}$, Tatsumi Koi ${ }^{7}$, \\ Jose Francisco Valdés-Galicia ${ }^{8}$, Luis Xavier González ${ }^{8}$, Ernesto Ortiz ${ }^{8}$, Octavio Musalem ${ }^{8}$, \\ Alejandro Hurtado ${ }^{8}$, Rocio Garcia ${ }^{8}$ and Marcos Anzorena ${ }^{8}$
}

\begin{abstract}
We plan to observe solar neutrons at Mt. Sierra Negra (4,600 m above sea level) in Mexico using the SciBar detector. This project is named the SciBar Cosmic Ray Telescope (SciCRT). The main aims of the SciCRT project are to observe solar neutrons to study the mechanism of ion acceleration on the surface of the sun and to monitor the anisotropy of galactic cosmic-ray muons. The SciBar detector, a fully active tracker, is composed of 14,848 scintillator bars, whose dimension is $300 \mathrm{~cm} \times 2.5 \mathrm{~cm} \times 1.3 \mathrm{~cm}$. The structure of the detector enables us to obtain the particle trajectory and its total deposited energy. This information is useful for the energy reconstruction of primary neutrons and particle identification. The total volume of the detector is $3.0 \mathrm{~m} \times 3.0 \mathrm{~m} \times 1.7 \mathrm{~m}$. Since this volume is much larger than the solar neutron telescope (SNT) in Mexico, the detection efficiency of the SciCRT for neutrons is highly enhanced. We performed the calibration of the SciCRT at Instituto Nacional de Astrofisica, Optica y Electronica (INAOE) located at 2,150 m above sea level in Mexico in 2012. We installed the SciCRT at Mt. Sierra Negra in April 2013 and calibrated this detector in May and August 2013. We started continuous observation in March 2014. In this paper, we report the detector performance as a solar neutron telescope and the current status of the SciCRT.
\end{abstract}

Keywords: Cosmic-ray; Solar neutron; Scintillator bar

\section{Findings}

\section{Introduction}

The particle acceleration mechanism on the solar surface has been studied through multi-wavelength observations. High-energy particles are produced related to solar flares. There are two kinds of particles accelerated at the time when a solar flare occurs, i.e., electrons and ions. Information of electron acceleration is obtained by hard X-ray, radio wave, and $\mathrm{H} \alpha$ bright line observations. On the other hand, very high-energy protons come to the earth and are sometimes observed. It is, however, difficult to understand the ion acceleration with the information of protons since

\footnotetext{
*Correspondence: sasaiyoshinori@stelab.nagoya-u.ac.jp

1 Solar-Terrestrial Environment Laboratory, Nagoya University, Furo-cho,

Chikusa-ku, Nagoya 464-8601, Japan

Full list of author information is available at the end of the article
}

they are affected by the interplanetary and geomagnetic fields. Gamma rays and neutrons produced by the interaction of accelerated ions and the solar atmosphere are sometimes used to study the ion acceleration as they are not affected by the magnetic field.

Ion acceleration has been mainly studied by gamma ray observation. The Reuven Ramaty High Energy Solar Spectroscopic Imager (RHESSI) was the first gamma ray imaging observatory for solar flares; its results revealed that there are differences between ion and electron acceleration foot point locations for several flares (Hurford et al. 2006). Furthermore, the Fermi Large Area Telescope (LAT) can observe high-energy gamma ray emission (>100 MeV) from the sun. Such high-energy gamma ray emission is generated from pion decay produced by the interaction between accelerated ions and the solar

\section{Springer}

(c) 2014 Sasai et al:- licensee Springer. This is an Open Access article distributed under the terms of the Creative Commons Attribution License (http://creativecommons.org/licenses/by/4.0), which permits unrestricted use, distribution, and reproduction in any medium, provided the original work is properly credited. 
atmosphere. Fermi LAT observed impulsive and long duration gamma ray emission on 7 March 2012 (Ajello et al. 2014).

Solar neutrons have been observed by the near-earth spacecrafts and ground-based detectors. Although solar neutrons are attenuated by the earth's atmosphere, highenergy neutrons ( $>100 \mathrm{MeV}$ ) may be detected by groundbased detectors. Solar neutron telescopes (SNTs) have been installed near the equator at high-altitude mountains; several solar neutron events were observed (Sako et al. 2006; Watanabe 2005). Since SNTs have a target scintillator for stopping neutron-induced proton and record counting rates when the energy of the recoil protons exceed the energy threshold level, it is not possible to record the energy of neutrons, event by event.

The SciBar Cosmic-Ray Telescope (SciCRT) project has been proposed as a brand new cosmic ray experiment using scintillator bars. The SciCRT is a multi-purpose cosmic ray detector. The main purpose of the SciCRT is 1) solar neutron observation for studying ion acceleration mechanism and 2) muon observation for investigating the anisotropy of Galactic Cosmic Rays (Kato et al. 2014; Nagashima et al. 2012). We performed the calibration of the SciCRT at Instituto Nacional de Astrofisica, Optica y Electronica (INAOE) in Mexico in 2012. The SciCRT was installed at Mt. Sierra Negra (4,600 m) in April 2013 and was calibrated in May and August 2013. In this paper, we will describe the performance of the SciCRT as a solar neutron detector.

\section{SciCRT detector}

The SciBar detector was initially developed for the K2K neutrino oscillation experiment in Japan (Yoshida et al. 1999). We can use this detector as a solar neutron telescope if we replace the direction of the accelerator beam by that of the sun. This new type of the solar neutron telescope has 1) a larger effective area than the current SNT, 2) enough energy resolution to determine the energy spectrum of solar neutrons with higher precision, 3) an ability to detect lower energy neutrons, and 4) particle identification ability.

Multianode photomultiplier tubes (MAPMTs) are attached to two sides, named ' $\mathrm{X}$ ' and ' $\mathrm{Y}$ '. A combination of two planes with 116 scintillator bars orthogonally attached makes 1 layer and there are 64 layers in total as shown in Figure 1. The top and bottom layers play a role in 1) four-fold coincidence planes of the muon detector and 2) anti-layers of solar neutron detector simultaneously. The top and bottom layers consist of the uppermost and bottommost two planes, each plane is named as upper and bottom X,Y. The other layers of the SciBar are longitudinally divided in eight components, each of which is called a super block (SB, see Figure 2). At the moment, we are

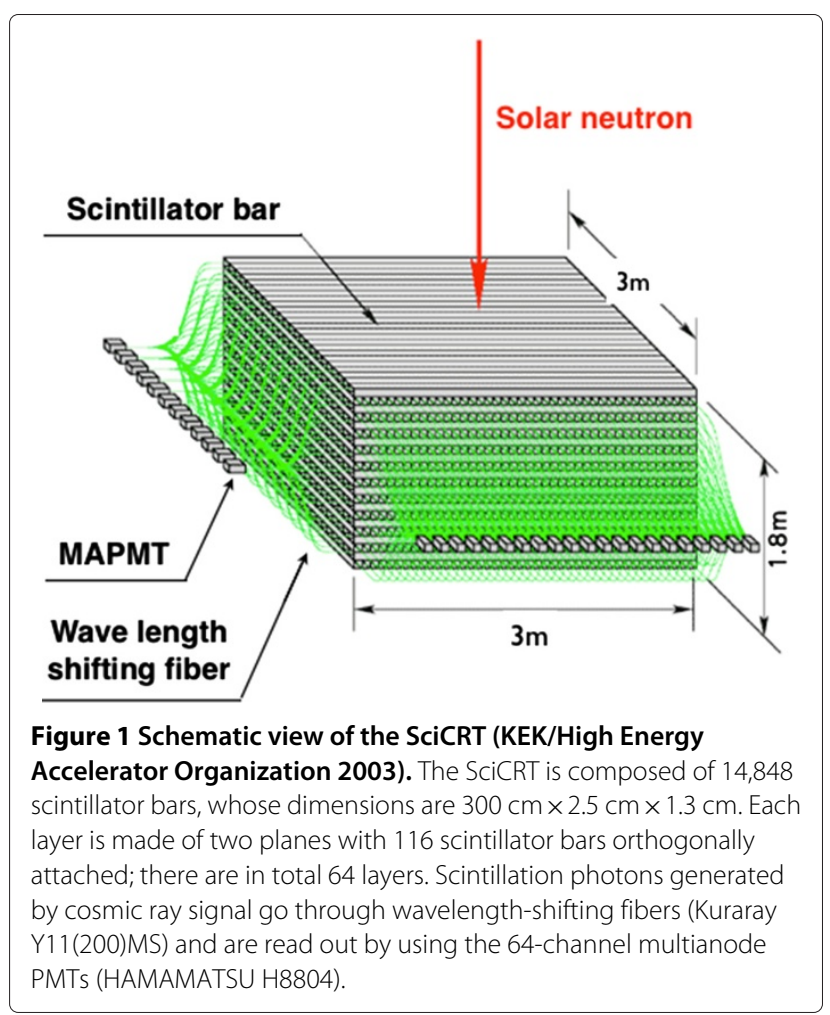

using $5 / 8$ of the full detector. We will improve this ratio in the near future.

\section{Data acquisition system}

For the time being, we use the data acquisition (DAQ) system developed for K2K experiment (Yoshida et al. 1999). There are three components configuring the DAQ system, i.e., front-end board (FEB), back-end board (BEB), and trigger board (TRGB). We will describe the flow of the DAQ system hereafter.

1) If more than 1 of the 32 channel signals from one MAPMT exceed the threshold level energy, a hit signal is generated on the FEB and sent to the TRGB via the BEB. 2) Several hit signals are collected on the TRGB. These signals are the test to decide if the neutron trigger condition is satisfied or not. A hold signal is generated if the trigger condition is satisfied. 3) The hold signal is sent to the FEB. The pulse height of each channel signal is kept until the hold signal reaches to the FEB. 4) The pulse heights are digitalized on the BEB. These digitalized values, named ADC data, have the deposited energy information at each channel. 5) ADC data is transferred to a DAQ personal computer (PC) via VME bus.

We need to keep the dead time in mind. Due to the limit of VME bus transfer, the transfer of ADC data is limited up to $1 \mathrm{kHz}$ per one BEB. We are now developing new fast readout electronics. We will describe our new electronics later. 


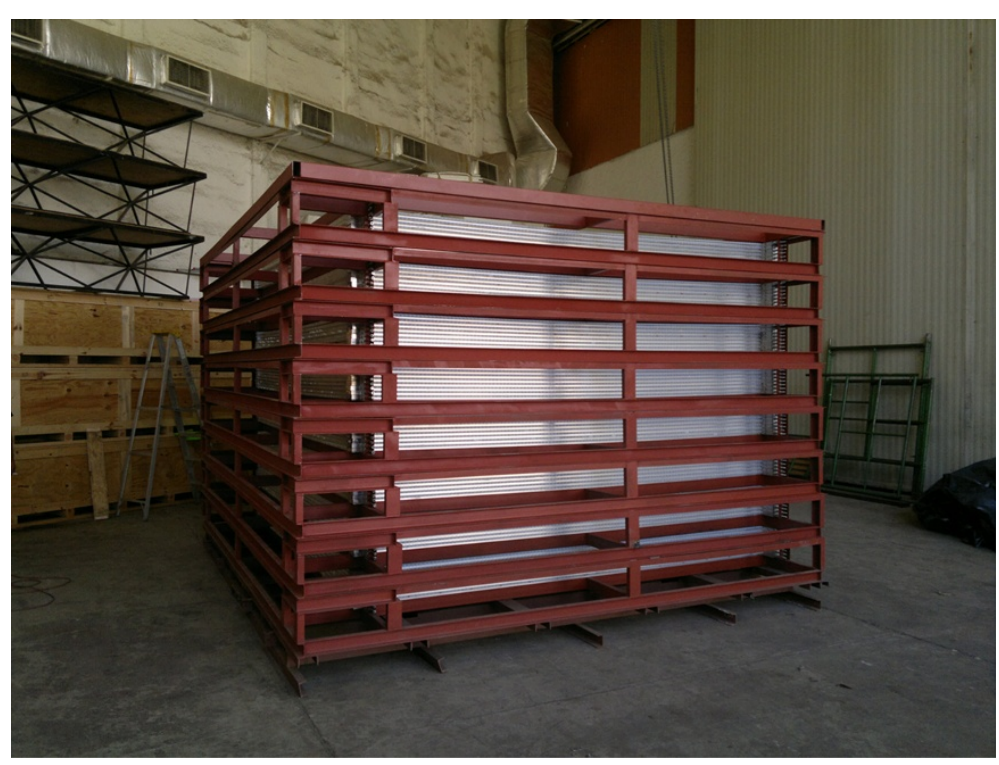

Figure 2 A picture of super blocks. A picture of the whole detector divided into eight super blocks (SBs). One SB is composed of 1/8 SciCRT supported by the steel-based structure to prevent the scintillator bars from deflecting by gravity.

The system of our DAQ hardware trigger is closed in each SB, since the trajectory of high-energy neutroninduced protons seldom penetrate over two SBs. This also mitigate the effect of dead time.

\section{Detection efficiency for neutrons}

To determine the detection efficiency of the SciCRT for neutrons, we estimated the effective area with each combination of incident angle and injected energy. Figure 3 shows the relationship between the incident energy of neutrons and the effective area. We can achieve a large effective area $(>50 \mathrm{MeV})$ for the SciCRT in comparison with that for the Mexico SNT (González et al. 2010). The detection efficiencies for neutrons by the SciCRT and the Mexico SNT are 10\% and 1\%, respectively, in case of $100 \mathrm{MeV}$ primary energy and $28 \%$ and $13.5 \%$ in case of $1 \mathrm{GeV}$ primary energy, respectively. As the incident energy becomes smaller, the difference in the efficiency becomes greater between the SciCRT and the SNT.

\section{Sensitivity as a solar neutron telescope}

The Mexico SNT is composed of proportional counters (anti counters) at the top, scintillation counters in the middle and proportional (directional) counters underneath. Scintillation counter consists of a 4- $\mathrm{m}^{2}$ array of 30$\mathrm{cm}$ thick plastic scintillator with four energy thresholds $(30,60,90,120 \mathrm{MeV})$. The Mexico SNT records the count rates with anti-coincidence for each threshold.

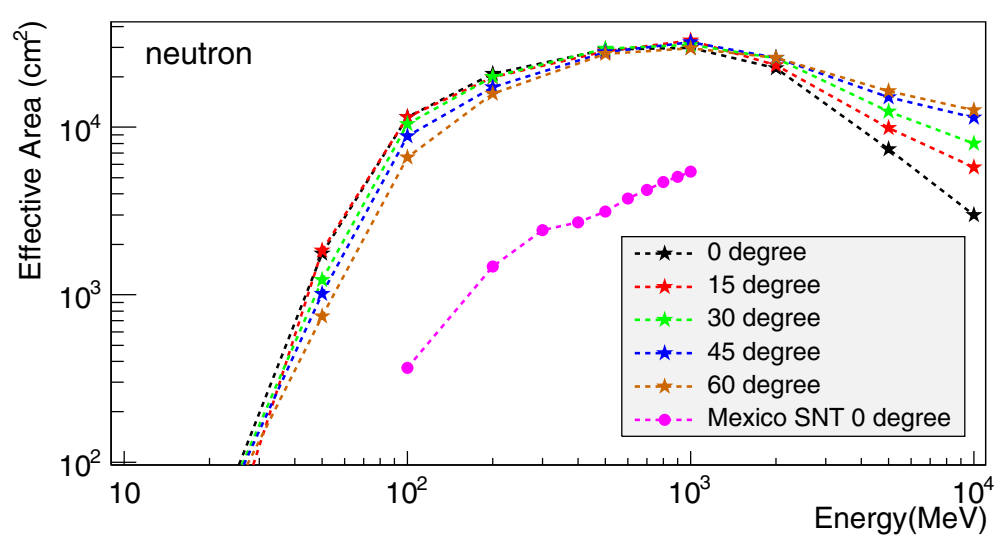

Figure 3 Detection efficiency for neutrons. Relationship between the energy of incident neutrons and the estimated effective area injected with five different zenith angles. The azimuth angle and injected direction are chosen randomly for each event. The pink plots show the effective area of vertical injection of particles on the Mexico SNT calculated by the detection efficiency (González et al. 2010) and detector size (4 m²) (Nagai et al. 2014). 


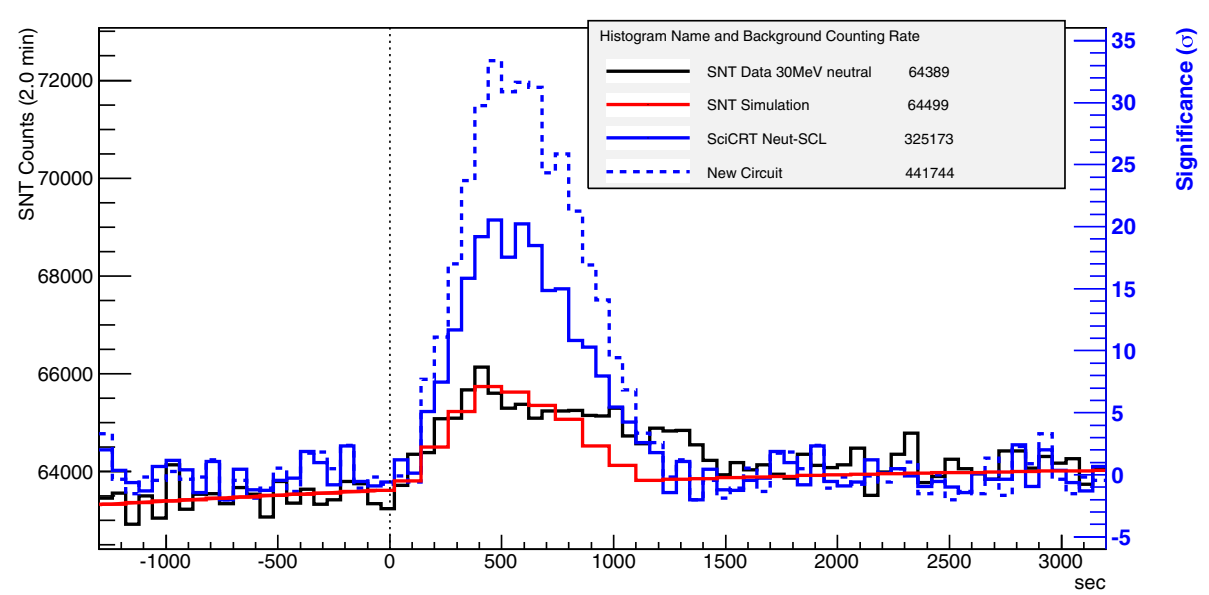

Figure $4 \mathrm{~A}$ time profile of the event on 7 September 2005. These are three kinds of time profiles from the solar neutron event on 7 September 2005, e.g., the MC calculation of the SciCRT, the data, and the MC calculation of the Mexico SNT. The Geotail hard X-ray peak time is 17:36:40 UT shown by a dot dash line. The vertical axis shows the count rates per 2 min on the left and the significance on the right. These vertical axis are normalized by the standard deviation calculated from background. The black line shows the data corresponds to the neutral channel ( $>30 \mathrm{MeV}$ ) on Mexico SNT. The red line is the best fit to the data assuming impulsive eruption of solar neutrons. These significance for the count rates per 5 min become 17, 32, and $53 \sigma$, respectively (Nagai et al. 2014).

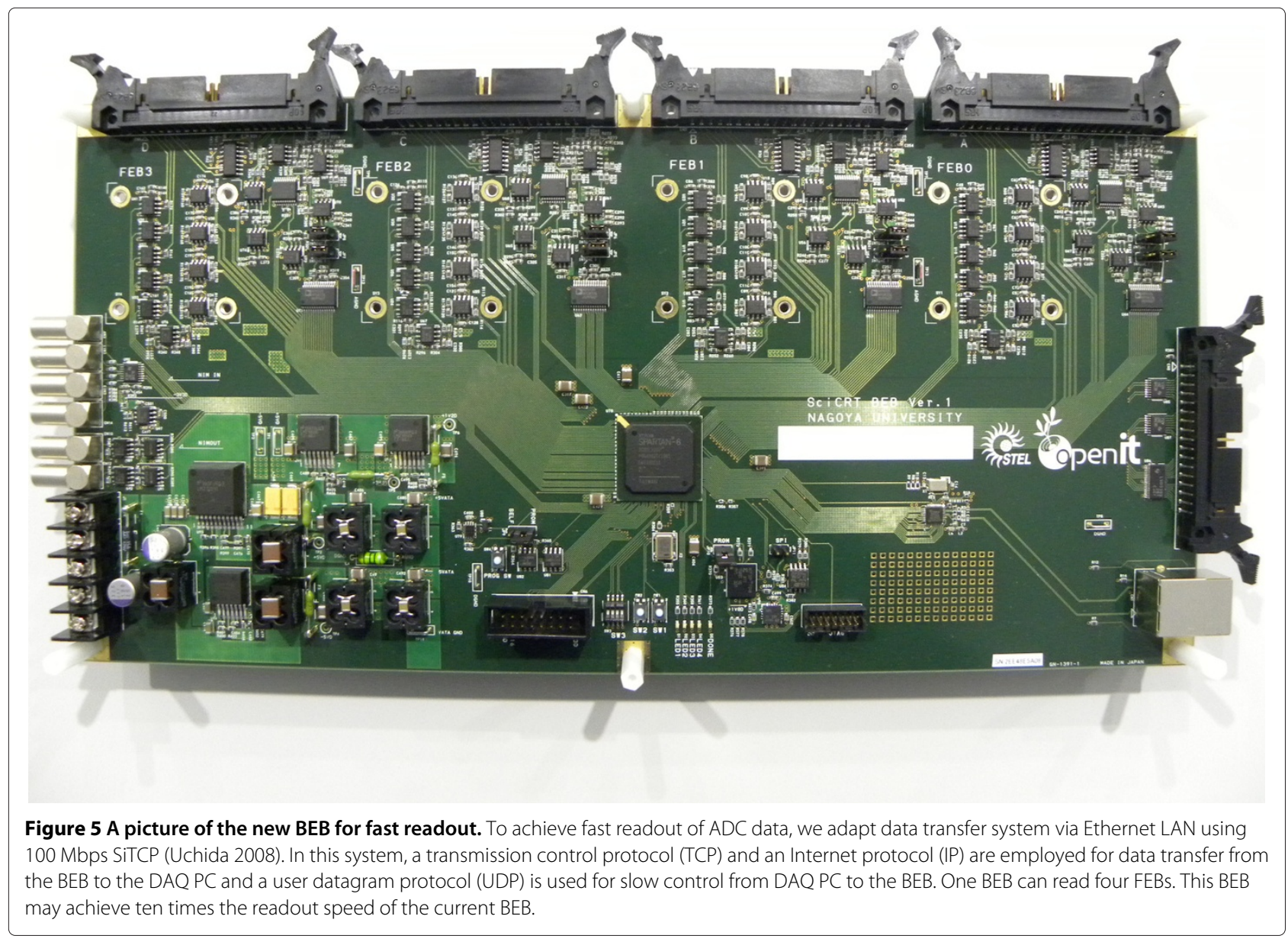



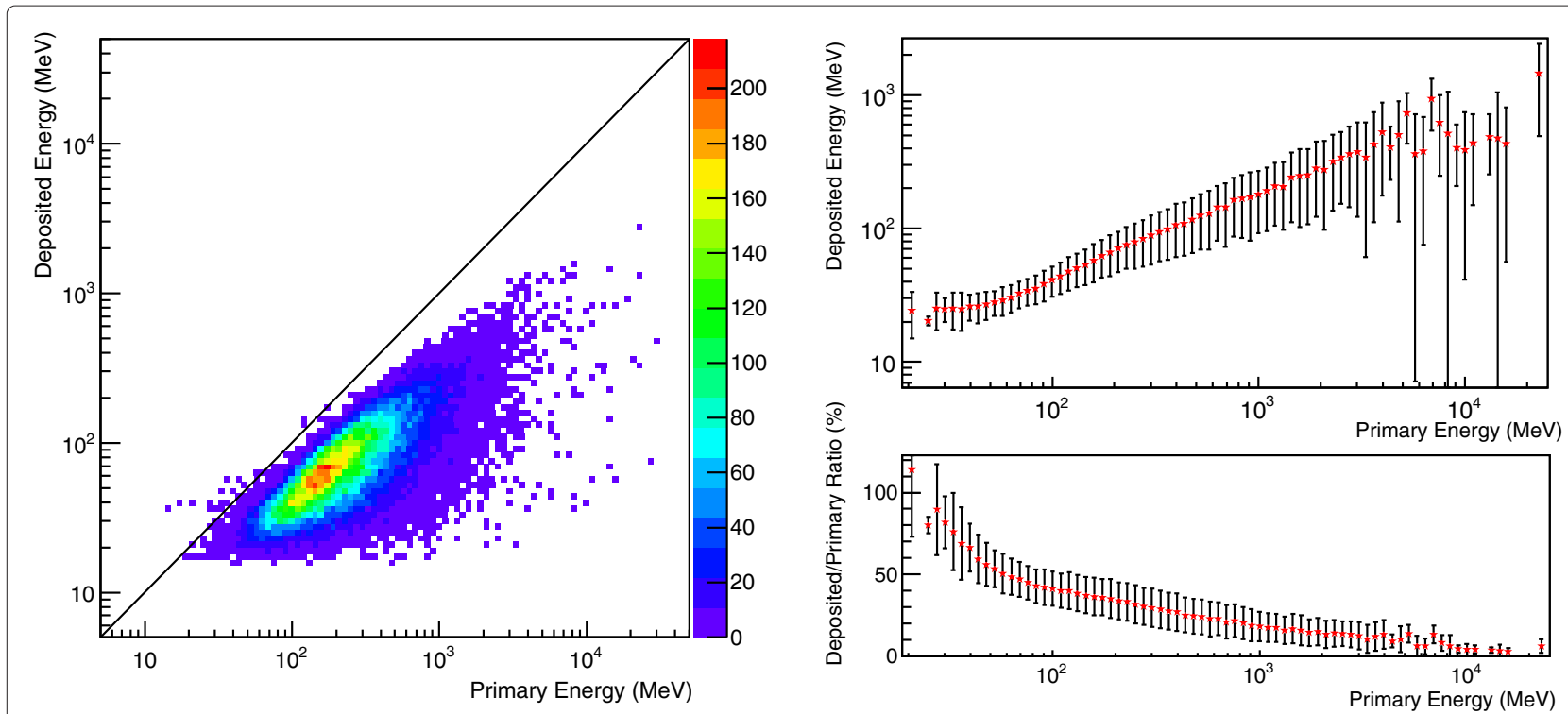

Figure 6 Relation between the primary neutron energy and neutron-induced proton energy. The left panel shows a correlation between the primary energy and the total deposited energy when background neutrons are injected to the detector. The black solid line means a linear function in case of completely reconstructed. The upper right panel represents the mean and the deviation of deposited energy for each injected. The lower right panel shows the ratio of deposited energy to injected (Nagai et al. 2014).

The SciCRT may also obtain the count rates with anticoincidence with a threshold $(7 \mathrm{MeV})$ determined by a MC calculation.

Figure 4 shows the time profile of neutrons triggered for the SciCRT obtained by a Monte Carlo (MC) simulation and for the Mexico SNT assuming the flux from the solar neutron event on 7 September 2005 (Sako et al. 2006). The parameters of the MC simulation are listed as follows. 1) The energy spectrum of neutrons at the sun is represented by a power law as $6.1 \times 10^{27}(\mathrm{E} / 100 \mathrm{MeV})^{-3.8} \mathrm{MeV}^{-1} \mathrm{sr}^{-1}$.
2) Neutrons are emitted impulsively at the sun. 3) The solar zenith angle is $17^{\circ} .5$. The propagation of solar neutrons through the earth's atmosphere is calculated using the Shibata program (Shibata 1994).

According to this calculation, the sensitivity of the SciCRT for neutrons is 1.9 times higher than that of the Mexico SNT. This sensitivity of the SciCRT for neutrons, however, is limited by the readout speed of the BEB developed for the neutrino oscillation experiment. Therefore, we are developing a fast readout BEB via Ethernet

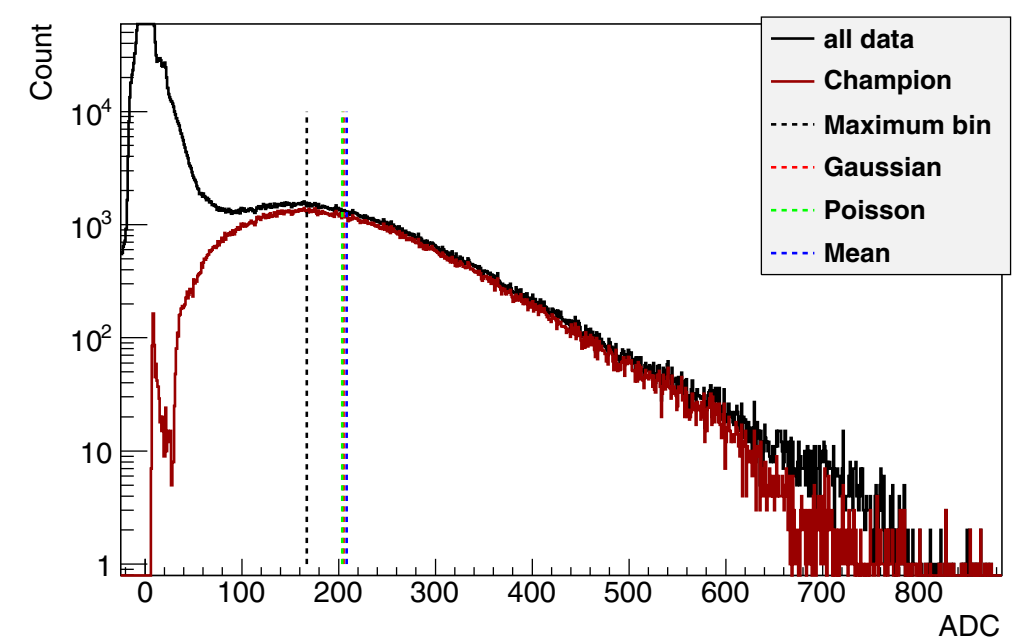

Figure 7 An example of the ADC histogram for muon/anti layers. The ADC histogram represents the data obtained by one of the MAPMTs at muon/anti layers. The black and red line show all data and 'Champion' histogram. The dotted lines show the result of the peak channels when fitting different analytical curves or simply finding the maximum in (see text) the histogram of Champion (Nagai 2014). 


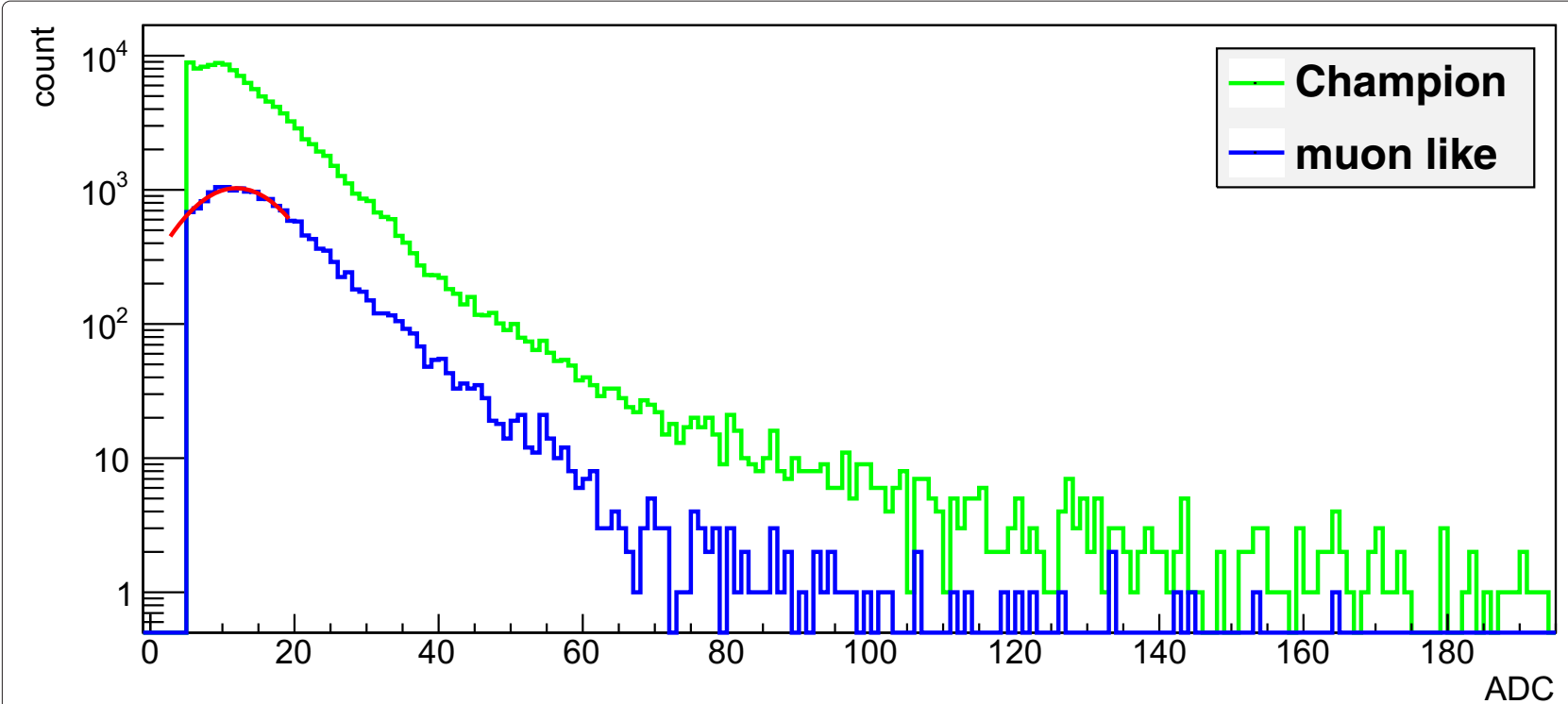

Figure 8 An example of the ADC histogram for neutron layers. This example shows the ADC histogram obtained by one of the MAPMTs for neutron layers. The green line shows the 'Champion' histogram. The blue line shows the histogram using the 'muon-like' criterion (Nagai 2014).

LAN (Sasai et al. 2013) (see Figure 5). We will achieve 3.1 times enhanced sensitivity than that obtained by the current SciCRT.

\section{Energy reconstruction}

Figure 6 represents the relationship between the total deposited energy and injected energy of background neutrons simulated by the Particle and Heavy Ion Transport code System (PHITS). It is possible to define the function to reconstruct the incident energy of neutrons from the total deposited energy in the scintillator bars (see the upper right panel), although the deposited energy measured is that of recoil proton.

\section{Observation at Mt. Sierra Negra}

The SciCRT started continuous observation at Mt. Sierra Negra in April 2014. For continuous observation, the gain and the threshold level must be adjusted to all MAPMTs. The gain is adjusted by changing voltages to the MAPMTs using the peak value of minimum ionization particle (MIP). The threshold level is controlled by the digital to analog converter (DAC) in the BEB. The requirements of

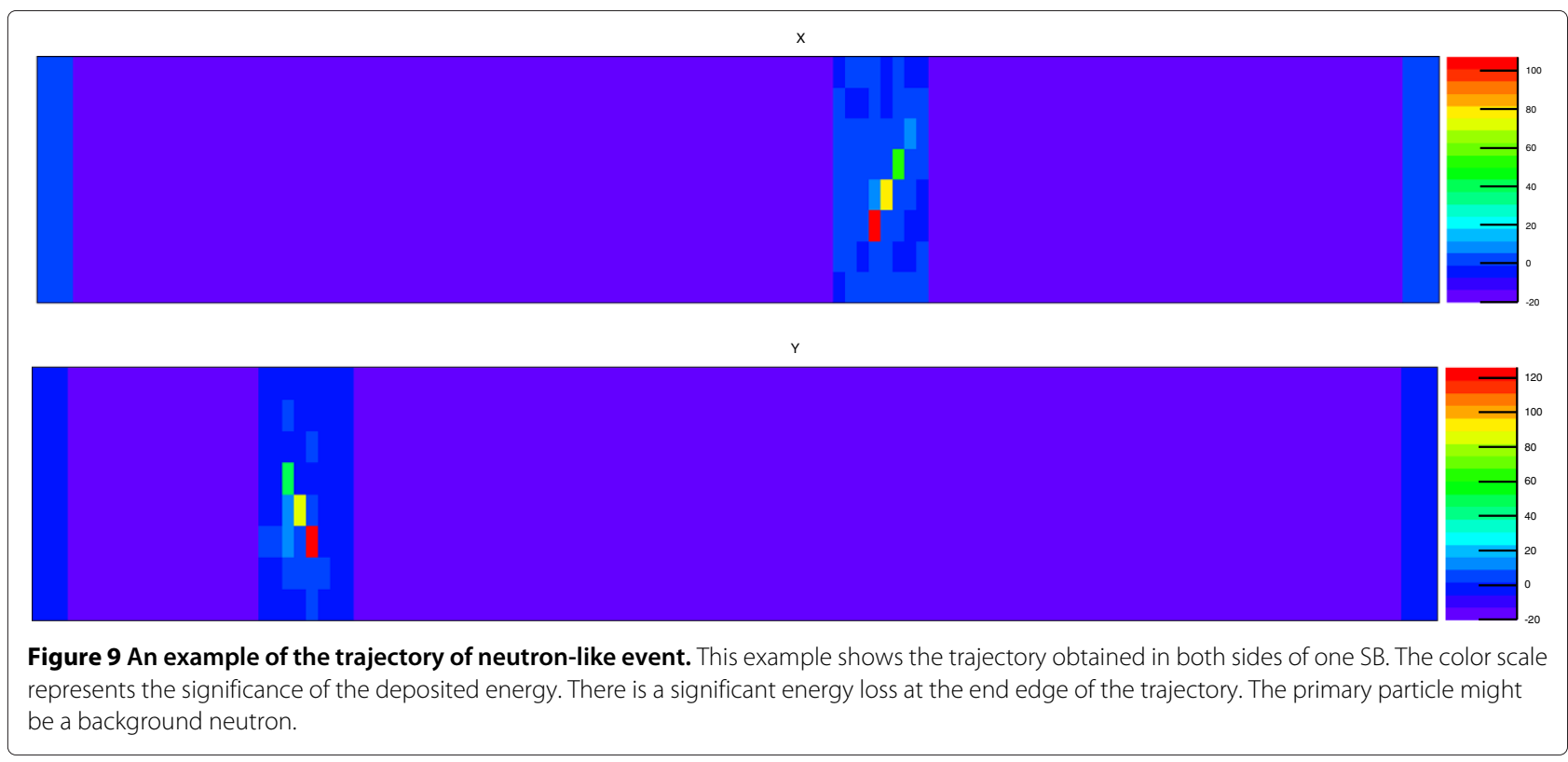


these values differ between muon/anti and neutron layers. The threshold is calculated by MC simulation, that the deposited energy becomes $7 \mathrm{MeV}$ for neutron layers and $0.3 \mathrm{MeV}$ for muon/anti layers. The peak of MIP and the threshold level for muon/anti layers are set to 300 and 70 ADC values, respectively. Those for neutron layers are set to 14 and $90 \mathrm{ADC}$ values. We calibrated the detector for background cosmic ray with muon trigger mode and obtained the ADC histogram to determine the gain and threshold.

There are 150 MAPMTs when we use $5 / 8$ of the full detector, each MAPMT has a different gain. We need to optimize supplied voltages by a simple method. We define 'Champion' as the channel which records the highest ADC value in one side of one horizontal plane for each cosmic ray event. Figure 7 shows the ADC histogram of one MAPMT for all data and Champion obtained by muon/anti layers. The peak of the ADC histogram is determined using four methods, i.e., maximum bin, Gaussian, Poisson and mean.

We accommodate this method to the neutron layers. However, since the required peak of MIP for neutron layers is very close to the pedestal histogram, it is difficult to separate between the pedestal and 'Champion' histogram. Therefore, it is necessary to adopt a criterion to select events. Due to selection of a muon-like event, Champion position of neutron layers is compared with that of muon/anti layers. The blue line shows the ADC distribution using this criterion in Figure 8.

Figure 9 shows the trajectory of a neutron-like event. Color at each pixel represents the relatively scaled deposited energy. Since the upper edge of this trajectory starts from the middle of the detector, it may be a trajectory of a neutron-induced proton. As this particle has a significant energy loss at the end of the trajectory, this may be the Bragg peak. This feature can be useful for particle identification, especially to discriminate a neutron from a gamma ray.

\section{Summary}

We installed the SciCRT as a multi-purpose cosmic ray detector at Mt. Sierra Negra $(4,600 \mathrm{~m})$ in eastern Mexico. We especially discussed its capabilities for solar neutron observation. The energy resolution of the SNTs and its sensitivity is not sufficient to elucidate the acceleration mechanism of ions.

The detection efficiency of the SciCRT for neutrons is estimated to be ten times higher than the Mexico SNT, especially at the low-energy region. The sensitivity of the SciCRT will be 1.9 times higher than the current Mexico SNT as shown by a Monte Carlo calculation. We are planning to upgrade electronics to resolve the inconveniently high dead time that cause a limited sensitivity of the SciCRT. The new electronics will improve 3.1 times the sensitivity compared with the Mexico SNT. Since the SciCRT is a fully active scintillator tracker, we can reconstruct the direction and deposited energy of every particle crossing through. We calculated the relationship between the primary neutron energy and neutron-induced proton energy and obtained the function to reconstruct the primary neutron energy. We expect to detect solar neutron events with the SciCRT during remaining solar cycle 24 .

\section{Competing interests}

The authors declare that they have no competing interests.

\section{Authors' contributions}

All authors read and approved the final manuscript.

\section{Acknowledgements}

We are grateful for the group of the SciBar and SciBooNe experiment for allowing us to use the SciBar as a cosmic ray detector. This work was supported by Grants-in-Aid for Scientific Research (B) 22340054, Scientific Research (C) 23540348 and Fellows 24340054 from JSPS. Grants from CONACYT (181879-I) and PAPIIT-UNAM (IN107911) are also acknowledged. This work is also supported in part by the joint research program of the Solar Terrestrial Environment Laboratory (STEL), Nagoya University.

\section{Author details}

${ }^{1}$ Solar-Terrestrial Environment Laboratory, Nagoya University, Furo-cho, Chikusa-ku, Nagoya 464-8601, Japan. ${ }^{2}$ Department of Physics, Shinshu University, Matsumoto 390-0802, Japan. ${ }^{3}$ College of Engineering, Chubu University, Kasugai 487-8501, Japan. ${ }^{4}$ Faculty of Engineering, Aichi Institute of Technology, Toyota 470-0392, Japan. ${ }^{5}$ Japan Atomic Energy Agency, Ibaraki 319-1184, Japan. ${ }^{6}$ Institute of Space and Astronautical Science, Japan Aerospace Exploration Agency, Yoshinodai 252-5210, Japan. ${ }^{7}$ SLAC National Accelerator Laboratory, Menlo Park, CA 94025, USA. ${ }^{8}$ Instituto de Geofisica, Universidad Nacional Autonoma de Mexico, Coyoacan 04510, D.F., Mexico.

Received: 2 May 2014 Accepted: 17 September 2014 Published: 30 September 2014

\section{References}

Ajello M, Albert A, Allafort A, Baldini L, Barbiellini G, Bastieri D, Bellazzini R, Bissaldi E, Bonamente E, Brandt TD, Bregeon J, Brigida M, Bruel P, Buehler R, Buson S, Caliandro GA, Cameron RA, Caraveo PA, Cecchi C, Chales E, Chekhtman A, Chiang J, Chiaro G, Ciprini S, Claus R, Cohen-Tanugi J, Cominsky LR, Conrad J, Cutini S, Ammando FD, et al. (2014) Impulsive and long duration high-energy gamma-ray emission from the very bright 2012 March 7 solar flares. The Astrophysical J 789:20

González LX, Sánchez FA, Valdés-Galicia JF, Medina-Tanco G (2010) Geant4 simulation of the solar neutron telescope at Sierra Negra, Mexico. Nuclear Instrum Methods Phys Res A 613:263-271

Hurford GJ, Krucker S, Lin RP, Schwartz RA, Share GH, Smith DM (2006) Gamma-ray imaging of the 2003 October/November solar flares. APJ 644:93-96

Kato C, Munakata K, Nakajima T, Kozai M, Itow Y, Matsubara Y, Sako T, Nagai Y, Sasai Y, Lopez D, Itow T, Mitsuka G, Shibata S, Takamaru H, Kojima H, Watanabe K, Tsuchiya H, Koi T, Ortiz E, Valdés-Galicia JF, Musalem O, Hurtado A, González LX, Garcia R, Anzorena M (2014) Initial performance of the SciCRT as a muon detector. Earth, Planets and Space. in press

KEK/high energy accelerator organization (2003). http://legacy.kek.jp/ newskek/2003/sepoct/K2KSciBar.html

Nagai Y (2014) Observation of cosmic rays by the new solar neutron telescope, SciCRT. PhD thesis, Department of Physics, Nagoya University

Nagai Y, Matsubara Y, Itow Y, Sako T, Lopez D, Sasai Y, Itow T, Munakata K, Kato C, Kozai M, Miyazaki T, Shibata S, Takamaru H, Kojima H, Tsuchiya H, Watanabe K, Koi T, Valdés-Galicia JF, Hurtado A, Musalem O, Ortiz E, González LX, Anzorena M, Garcia R (2014) First cosmic-ray measurements by the scicrt solar neutron experiment in mexico. Astroparticle Phys 59:39-46 
Nagashima K, Morishita I, Fujii Z, Mori S, Kondo I, Jacklyn RM (2012) The existence of cosmic ray sidereal anisotropies of galactic and solar origins with energies lower than $104 \mathrm{gev}$ and their modulation caused by the presumed behavior pattern of the heliomagnetosphere and of its neighboring gaseous matter in interstellar magnetic field. Earth Planets Space 64:613-639

Sako T, Watanabe K, Muraki Y, Matsubara Y, Tsujihara H, Yamashita M, Sakai T, Shibata S, Valdés-Galicia JF, González LX, Hurtado A, Musalem O, Miranda P, Martinic N, Ticona R, Velarde A, Kakimoto F, Ogio S, Tsunesada Y, Tokuno H, Tanaka YT, Yoshikawa I, Terasawa T, Saito Y, Mukai T, Gros M (2006) Long-lived solar neutron emission in comparison with electron-produced radiation in the 2005 september 7 solar flare. APJ 651:69-72

Sasai Y, Kawabata T, Itow Y, Matsubara Y, Sako T, Nagai Y, Lopez D, Itow T, Mitsuka G, Munakata K, Kato C, Yasue S, Kozai M, Nakano Y, Miyazaki T, Shibata S, Takamaru H, Kojima H, Tsuchiya H, Watanabe K, Koi T,

Valdés-Galicia JF, Hurtado A, Musalem O, Ortiz E, González LX, Anzorena M, Garcia R (2013) Development of a fast data taking system for a new cosmic ray detector (SciCRT) at Mt. Sierra Negra, Mexico. To appear in the Proceedings of the 33rd International Cosmic Ray Conference, Rio de Janeiro, Brazil, 2013

Shibata S (1994) Propagation of solar neutrons through the atmosphere of the earth. J Geophys Res 99:6651-6665

Uchida T (2008) Hardware-based TCP processor for Gigabit Ethernet. IEEE Trans Nuclear Sci 55:1631-1637

Watanabe K (2005) Solar neutron events associated with large solar flares in solar cycle 23. PhD thesis, Department of Physics, Nagoya University

Yoshida M, Yamamoto S, Murakami T, Tanaka M, Nakaya T, Nishikawa K, Joo KK, Kim BJ, Kim JY, Kim SB, Lee MJ, Lim IT (1999) Development of the readout system for the K2K SciBar detector. IEEE Trans Nuclear Sci 51:3043-3046

doi:10.1186/1880-5981-66-130

Cite this article as: Sasai et al:: Performance of the SciBar cosmic ray

telescope (SciCRT) toward the detection of high-energy solar neutrons in solar cycle 24. Earth, Planets and Space 2014 66:130.

\section{Submit your manuscript to a SpringerOpen ${ }^{\circ}$ journal and benefit from:}

- Convenient online submission

- Rigorous peer review

- Immediate publication on acceptance

- Open access: articles freely available online

- High visibility within the field

- Retaining the copyright to your article

Submit your next manuscript at $\boldsymbol{\nabla}$ springeropen.com 